\title{
Formation and arrangement of pits by a corrosive gas
}

\author{
James Burridge \\ Department of Mathematics, University of Portsmouth, Portsmouth PO1 3HF, United Kingdom \\ Robert Inkpen \\ Department of Geography, University of Portsmouth, Portsmouth PO1 3HE, United Kingdom \\ (Received 26 September 2014; revised manuscript received 16 December 2014; published 6 February 2015)
}

\begin{abstract}
When corroding or otherwise aggressive particles are incident on a surface, pits can form. For example, under certain circumstances rock surfaces that are exposed to salts can form regular tessellating patterns of pits known as "tafoni." We introduce a simple lattice model in which a gas of corrosive particles, described by a discrete, biased diffusion equation, drifts onto a surface. Each gas particle has a fixed probability of being absorbed and causing damage at each contact. The surface is represented by a lattice of strength numbers which reduce after each absorbtion event, with sites being removed when their strength becomes negative. Regular formations of pits arise spontaneously, with each pit having a characteristic trapezoidal geometry determined by the particle bias, absorbtion probability, and surface strength. The formation of this geometry may be understood in terms of a first order partial differential equation and is a consequence of particle concentration gradients which arise in the pits. By viewing pits as particle funnels, we are able to relate the gradient of pit walls to absorbtion probability and particle bias.
\end{abstract}

DOI: 10.1103/PhysRevE.91.022403

PACS number(s): 82.65.+r, 34.35.+a, 05.10.Gg, 89.75.Kd

\section{INTRODUCTION}

Pits forming in clusters on the surfaces of rocks have been studied for over a century [1]. Pitting corrosion is also one of the major damage mechanisms in metals and other materials used in engineering structures [2]. In both rock and metal, the processes which lead to pit formation involve multiple physical phenomena. They have in common that corrosive or otherwise aggressive material must be transported onto the surface. With this in mind, we investigate pit formation using the simplest possible model that includes a transport process, and for simplicity we refer to the damage caused by particles, which might, in practice, not be chemical, as corrosion. Our aim is to discover what structures are formed on a surface when corrosive or otherwise aggressive particles are biased toward it.

Pit formation is of importance in both geology and engineering. For example, geologists are interested in rock forms created by regular clustering of pits. These occur in many climatic zones including coasts and deserts, and in different lithologies [1,3]. An example is shown in Fig. 1. Such formations have been labeled as "cavernous," "alveolar," "honeycomb," and, most commonly, "tafoni." We adopt this latter term, the plural of "tafone," a single pit. There is a general consensus that they are created by salt weathering $[1,4,5]$, although some chemical weathering processes [6] and biological agents [7] have been suggested as being significant. Salt crystallization and expansion produce stresses in the rock that result in erosion but why regular pits are spontaneously formed rather than merely surface lowering is not yet clear $[3,8]$. Recent work has also highlighted the importance of a rock's characteristics, for example, its strength, porosity, or surface harness, as controlling factors $[9,10]$.

Theories for the mechanism driving tafoni growth include hardening of the top layer of rock [11], softening of the rock core by chemical processes [12], and wind acceleration in the cavity [13]. However, it has been noted that there is no generally agreed upon classification of the forms that tafoni can take, or of the formation processes [3,8], many of which have yet to be modeled mathematically. One proposed mechanism that has been modeled mathematically is the evolution of a single pit driven by the migration and crystallization of salts due to cycles of wetting and drying [14]. Single tafone are theoretically shown to grow as a result of excess salt crystallization in regions with low evaporation rates, deeper in the pits. In addition to this physical model, a phenomenological approach has been introduced [15], where a simple functional form is hypothesized for the relationship between the rate at which pits deepen and their age. The form is motivated by viewing the development of tafoni as a process involving positive and negative feedback between pit shape and growth rate. Deepening occurs rapidly at first before reaching a critical depth, after which it slows. It appears that no mathematical model exists in which the regular formations of pits seen in nature (see Fig. 1) arise spontaneously.

In engineering applications, pit corrosion in metals is one of the most difficult corrosion mechanisms to manage. Perhaps due to its economic importance, metal pitting has been the subject of a great deal more mathematical research than the formation of tafoni. Pits in metal surfaces are believed to propagate due to the concentration of chlorides inside them [2]. Pits originate as tiny irregular nucleation sites, which can then either stop growing or propagate rapidly [2]. Lattice gas cellular automata are commonly used to model pit formation [16-18], and these models have captured the initiation and propagation of pits; however, analytical results on pit geometry or growth rate have yet to be found.

In our model we see that pits form when fluctuations in the corrosion process create depressions of sufficient depth to collect proportionally more corrosive particles than neighboring regions. These collected particles then cause the depression to deepen relative to the rest of the surface forming a pit. Despite its simplicity, the model shares a common feature with the wetting and drying process [14] 


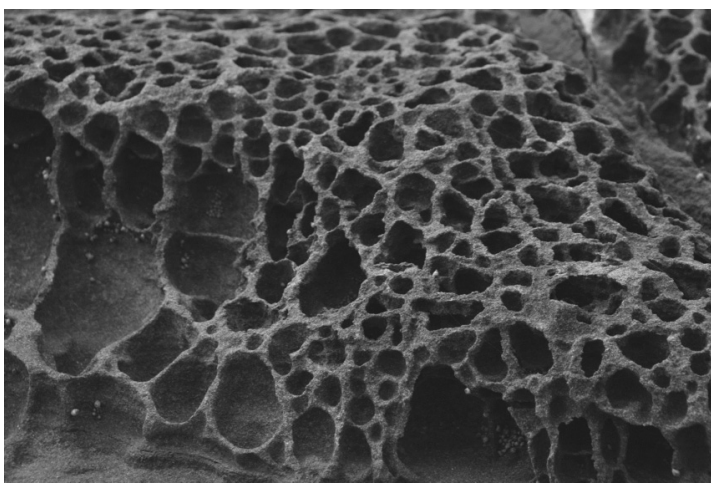

FIG. 1. Image of tafoni from Louttit Bay, near Lorne, Victoria, Australia.

and the phenomenological model [15] in that pit growth is a consequence of positive feedback between shape and growth rate: Deeper pits initially grow faster. We also see that pits reach a stable depth, mimicking the negative feedback reported phenomenologically. In addition, as we will see, the model spontaneously produces clusters of pits creating formations of striking regularity. The model is not a complete description of any particular pitting phenomenon. However, its simplicity and tractability permit us to gain insight into how regular pits can form, in general.

\section{THE MODEL}

Our model is defined in discrete time on an integer lattice, the sites of which can be either gas or solid. The interface between the two regions defines the surface of the solid. We consider lattices of two and three dimensions corresponding to surfaces of dimensions one or two, but our main focus is on one dimensional surfaces. In common with studies of pitting in metals $[16,18]$, we begin by considering a simple model of surface corrosion by discrete particles. In the gas region these perform random walks biased in the direction of the surface. This mimics, in the case of salt weathering of rocks, salt particles being carried onto a rock surface by wind or sea spray. Initially, all solid sites are given a strength number. If a corrosive particle occupies a site adjacent to the surface and its next randomly chosen step would take it into the surface, it is absorbed with probability $p_{c}$, the "corrosion probability." Otherwise, it remains in its current position. Absorbtion of a particle reduces the strength of the surface site by a fixed quantity. The process is illustrated in Fig. 2.

Although our model is based on the process just described, rather than modeling individual particles, we model the density of a population of particles. In doing so we neglect fluctuations in the corrosion rate over the surface which arise from the discrete nature of the particles. These fluctuations would have the effect of roughening the surface on short length scales, with regular pit formations dominating at larger scales, preventing the formation of a fractal surface [19]. We arrive at an evolution equation for the particle density by noting that the probability mass function for a single random walking particle evolves according to a discrete time and space master equation [20] with surface boundary condition corresponding to an absorbtion probability $p_{c}$. If we neglect collisions

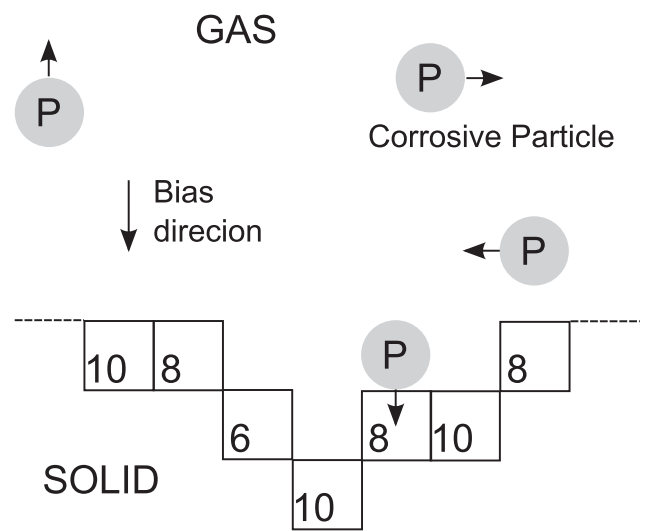

FIG. 2. Schematic representation of the corrosion process on a two dimensional lattice. The numbers in the surface of the solid matrix represent the strength of the solid in that location. The numbers are reduced when a corrosive particle is absorbed by the surface. The motion of corrosive particles is biased toward the surface.

and exclusion constraints between particles, then the master equation also describes the evolution of the density of a population of particles. If $\phi(\vec{x}, t)$ is the particle density at gas site $\vec{x}$ at time $t$, then this evolution equation is

$$
\begin{aligned}
\phi(\vec{x}, t+1)= & \phi(\vec{x}, t)+\sum_{\vec{y} \in G(\vec{x})} p(\vec{y} \rightarrow \vec{x}) \phi(\vec{y}, t) \\
& -\sum_{\vec{y} \in G(\vec{x})} p(\vec{x} \rightarrow \vec{y}) \phi(\vec{x}, t) \\
& -p_{c} \sum_{\vec{y} \in B(\vec{x})} p(\vec{x} \rightarrow \vec{y}) \phi(\vec{x}, t),
\end{aligned}
$$

where $G(\vec{x})$ is the set of nearest neighbor gas sites of $\vec{x}$ and $B(\vec{x})$ is the set of nearest neighbor solid sites of $\vec{x}$. The transition probabilities are given by

$$
p(\vec{x} \rightarrow \vec{y})=\frac{1}{d} \begin{cases}b, & \text { if } \vec{x} \text { above } \vec{y}, \\ 1-b, & \text { if } \vec{x} \text { below } \vec{y}, \\ \frac{1}{2}, & \text { otherwise, }\end{cases}
$$

with $d$ the dimension of the lattice and $b \in[0,1]$ a bias. Equations (1) and (2) define a discrete equivalent of the biased diffusion equation [20].

At each time step, the strength of each surface site is reduced in magnitude by the particle density it absorbs. This corrosion rule, together with Eq. (1) and the initial distribution of solid site strengths, defines our model. Throughout this paper we assume that the initial strength of each site is a random variable uniform on $[(1-\xi) S,(1+\xi) S]$ and independent of the strength values of its neighbors. The number $S$ is mean site strength, and $\xi$ is referred to as the "noise." The variance of site strength is $(\xi S)^{2} / 3$. The corrosive damage per unit particle density may be varied by altering $S$.

\section{NUMERICAL RESULTS}

Equation (1) must be solved numerically because of the complexity of the surface once a few surface sites have been destroyed. We consider a finite system and impose lateral periodic boundary conditions and set the particle density at a 
fixed distance, $h_{G}$, above the highest point of surface equal to a constant $\rho>0$. The height $h_{G}$ is chosen sufficiently large that further increase would not affect the particle density near the surface.

\section{A. One dimensional surfaces}

We consider an initially level surface so that prior to the destruction of the first surface site, the particle density is equal along the interface. In the absence of any noise $(\xi=0)$, all surface sites are simultaneously destroyed, preserving the flat interface, one unit lower. Due to the definition of the height, $h_{G}$, of the gas domain, the equilibrium particle density at each point of the interface depends only on its level relative to other interface sites. Therefore, the corrosion process wears away a flat surface at a constant rate over time.

In the case $\xi>0$, small pits in the surface appear, as observed in metals [2]. Their evolution depends on two effects. First, sites at their edges will have two faces exposed to the gas and therefore erode more quickly, widening the pit and smoothing the surface. The second effect arises from the on-surface bias of the gas particles, which, provided $p_{c}$ is sufficiently small, causes particles to visit the bases of the pits more frequently, deepening them. Together, these effects cause small fluctuations in the interface to evolve toward larger, smoother pits. The early stages of this process are illustrated in Fig. 3. These early fluctuations in surface depth lack order. Over a longer period, for example, in Fig. 4, the surface evolves toward a stable state characterized by regular trapezoidal pits.

If $p_{c}$ is too large, then the pit deepening effect will vanish because particles are not able to sufficiently explore the surface and find its deepest parts before they are absorbed. This gives rise to a depletion zone in the particle density above the surface.

In Fig. 5 we show the results of varying $p_{c}$ continuously across the system so that a distance $x$ from the left boundary of a system of width $W$, the corrosion probability follows a triangular distribution, $p_{c}(x)=\max \left(p_{c}\right)+\left[\max \left(p_{c}\right)-\min \left(p_{c}\right)\right] \mid$ $2 x-W \mid / W$, with its maximum at $x=W / 2$. With lower

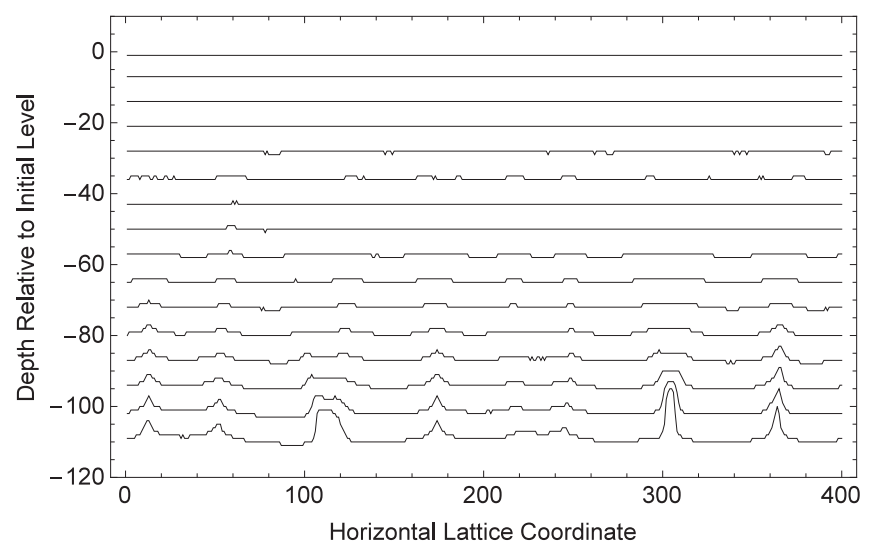

FIG. 3. The early evolution of surface fluctuations in the $d=2$ lattice model. The rock surface depth, relative to its starting value, is plotted at 1000 time step intervals. The model parameters in this case are $S=10, \xi=0.05, b=0.58, p_{c}=0.025, \rho=1$.

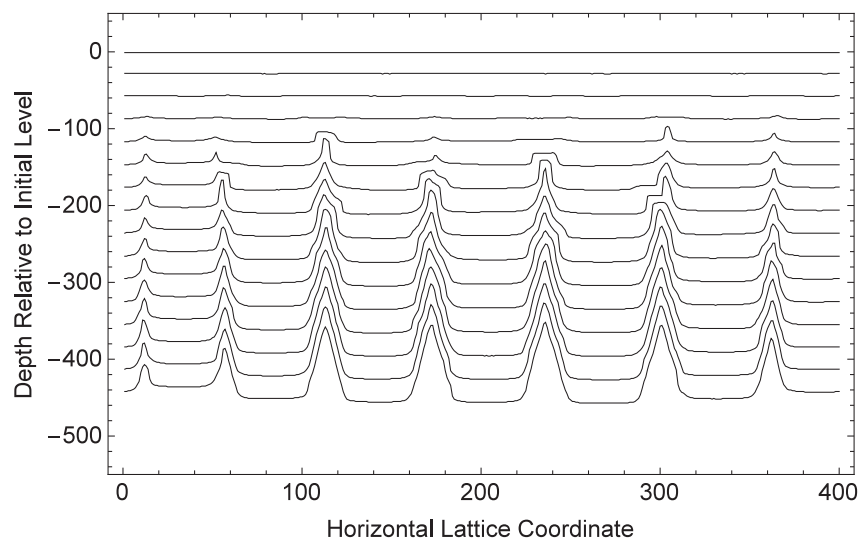

FIG. 4. The spontaneous evolution of a regular arrangement of pits in the $d=2$ lattice model. The interface sequence is drawn from the same simulation as Fig. 3 but plotted at 4000 time step intervals.

corrosion probability, particles are less likely to be absorbed by pit walls and more likely to drift down to corrode the base, resulting in deeper and narrower structures.

The magnitude of the noise parameter, $\xi$, influences the early stages of the process and, although it does not influence the gradient of pit walls, larger noise values roughen the regular trapezoidal formations and can create narrower pits, which later merge. A smaller noise value increases the time taken for fluctuations to develop from the flat surface, and for given values of $b, p_{c}$ there appears to be a critical value of $\xi$, below which pits fail to emerge. In this work we focus on the case where $\xi$ is sufficiently large for pits to develop but not large enough to create fluctuations which disrupt regular formations.

\section{B. Dynamics of pit depth}

From Figs. 3 and 4 we see that at least $10^{4}$ time steps pass before the initially flat surface begins to develop sufficient fluctuations for pit development to begin. These "protopits"

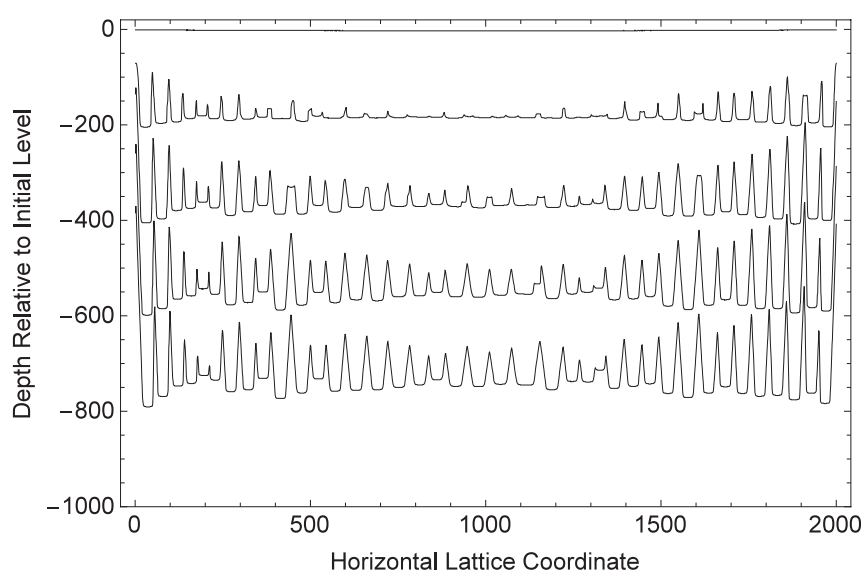

FIG. 5. The spontaneous evolution of a regular arrangement of pits in the $d=2$ lattice model with variable corrosion probability. The rock surface depth, relative to its starting value, is plotted at 20000 time step intervals. The model parameters in this case are $S=10, \xi=$ $0.05, b=0.6, \rho=1$, with $p_{c}$ varying piecewise linearly from $p_{c}=$ 0.01 at the boundaries to $p_{c}=0.05$ in the center of the system. 


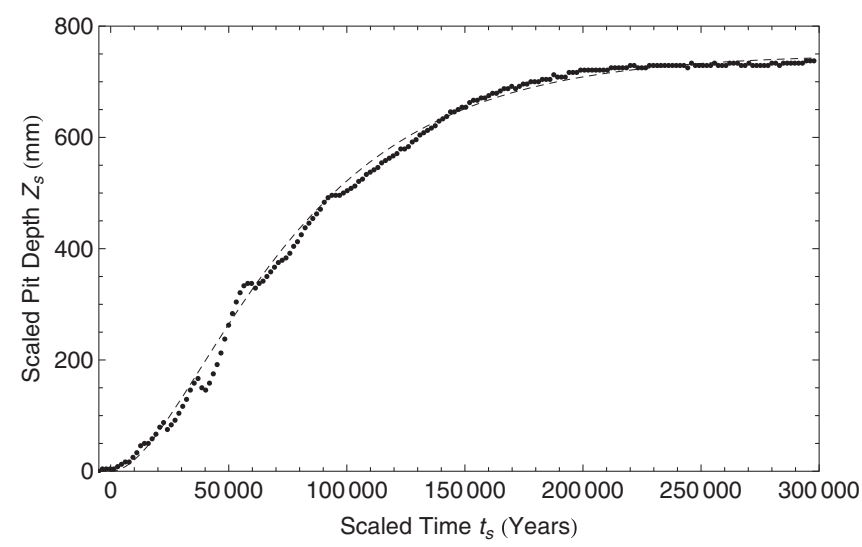

FIG. 6. Evolution of pit depth (measured by difference between maximum and minimum height of surface) in the $d=2$ lattice model. For comparison with depth data for sandstone tafoni in northeastern Arizona [15], the simulated depth $(D)$ and simulation time $(T)$ have been scaled as follows: $t_{s}=0.324 T-10^{4}$ and $Z_{s}=4.17 D$ to match the field data in units of $\mathrm{mm}$ and years. The dashed line shows the growth curve (3) derived from the field data, having parameter values (determined by Sunamura and Aoki [15]) $n=0.89, \beta=0.0000177$, and $Z_{c}=750.1$. The model parameters are $S=10, \xi=0.05, b=$ $0.58, p_{c}=0.025, \rho=1$, as in Fig. 4 .

initially deepen slowly and then accelerate before reaching their equilibrium depth, as shown in Fig. 6. This behavior matches field observations of tafoni growth rates [3,15,21]. To provide a quantitative comparison to field data, in Fig. 6 we also have a graph of the phenomenological growth curve proposed by Sunamura and Aoki [15],

$$
Z(t)=Z_{c}\left[1-(n+1) e^{-\beta t}+n e^{-\left(1+n^{-1}\right) \beta t}\right],
$$

where $Z(t)$ is pit depth at time $t$ (years), $Z_{c}$ is final depth, and $n, \beta$ are constants which capture the shape of the growth curve and the growth rate. Whereas $n$ is dimensionless, $\beta$ has the dimension of inverse time and is proportional to the maximum rate of increase of pit depth. To make a direct comparison between our model and field data, in Fig. 6 these parameters are set equal to values obtained by Sunamura and Aoki [15] for sandstone tafoni found in northeastern Arizona, and the simulation data are scaled to match the stable depth and the time taken to reach it. We note that although the "S-shaped" growth curve is believed to be universal, the values of parameter $\beta$, calibrated from field observations by Sunamura and Aoki [15], lie in a wide range, $8.9 \times 10^{-6}<$ $\beta<3.5 \times 10^{-2}$, depending on the geographical location and rock type, reflecting wide variations in the time taken for tafoni to reach their final depth. The parameter $n$ falls in a much narrower range, $0.01<n<1$, with larger values producing an initially slower growth rate and therefore a more pronounced S shape to the growth curve. From Fig. 6 we see that our simulation results capture this $\mathrm{S}$ shape, and the model gives some insight into the feedback mechanisms behind the growth process $[3,15,21]$. We have shown that growth is slow at first while pits take time to emerge from random fluctuations. It then accelerates as pits funnel particles into their bases, evolving toward a trapezoidal equilibrium which

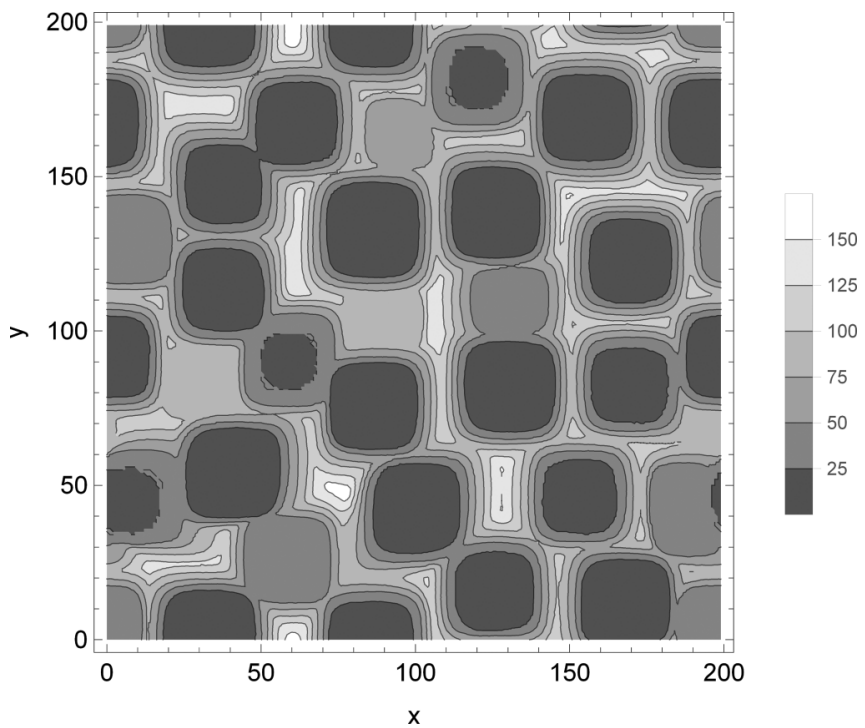

FIG. 7. Contour plot of spontaneously evolved pits in the $d=3$ lattice model after 25000 time steps. Here $x$ and $y$ are horizontal lattice coordinates and contours are plotted at 25 lattice unit intervals with zero corresponding to the deepest point on the surface. The model parameters in this case are $S=5, \xi=0.2, b=0.6, p_{c}=0.02$.

finally ceases to deepen due to the presence of neighboring pits.

\section{Two dimensional surfaces}

When the corrosive particles are incident on a two dimensional interface we again find that early random fluctuations in the interface due to differential site strength evolve toward regular pit structures. As Fig. 7 shows the regularity in spatial arrangement is less pronounced than for the one dimensional interface. However, a cross section through the surface (Fig. 8) shows that very similar trapezoidal pit shapes are present. As with the one dimensional case, a lower on-surface bias (see Fig. 9) produces wider, shallower pits. This effect appears to be independent of dimension.

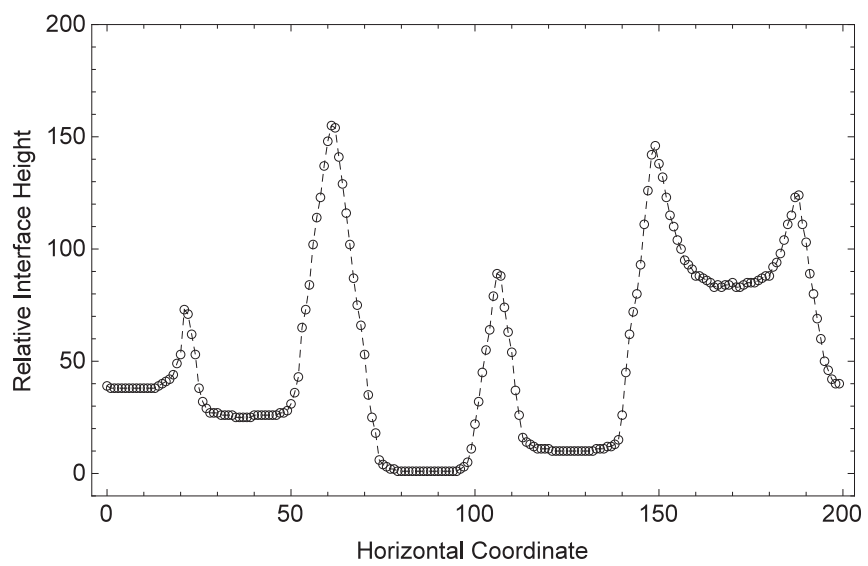

FIG. 8. Cross section through surface in the $d=3$ lattice model. The model parameters in this case are $S=5, \xi=0.2, b=0.6, p_{c}=$ 0.02 (identical to Fig. 7). 


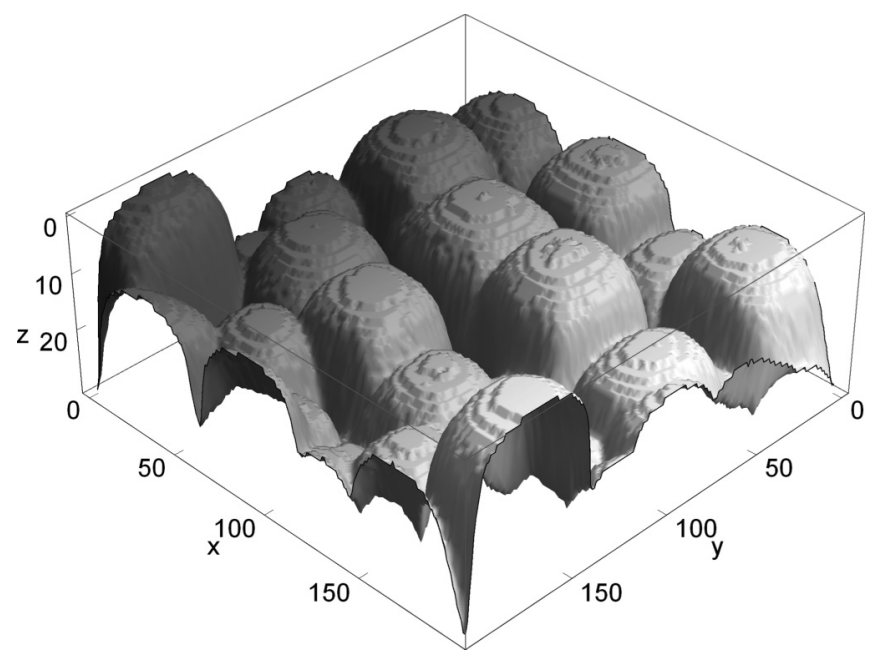

FIG. 9. Underside view of spontaneously evolved pits in the $d=$ 3 lattice model. Here $x$ and $y$ are horizontal lattice coordinates and $z$ measures height (in lattice units) relative to the deepest point on the surface. The model parameters in this case are $S=5, \xi=0.2, b=$ $0.55, p_{c}=0.02$. Note that pits are wider and shallower compared to Fig. 7 and 8 due to lower on-surface bias.

\section{CORROSION GRADIENT ANALYSIS}

We now analyze the corrosion process when the corrosion rate, equivalent to particle density adjacent to the pit walls, is defined externally to the model. The complex interactions between particle flow and surface morphology are replaced with a simple functional relationship between corrosion rate and depth. The relationship is determined by observing the particle density in a spontaneously formed pit in the full model. Figure 10 shows that adjacent to the upper walls the density is approximately constant and increases exponentially toward the base. Also shown is a fitted function of the form $A+B \times$ $\beta^{h}$. Since this functional form accurately captures the particle density adjacent to the wall and because the evolution of pit

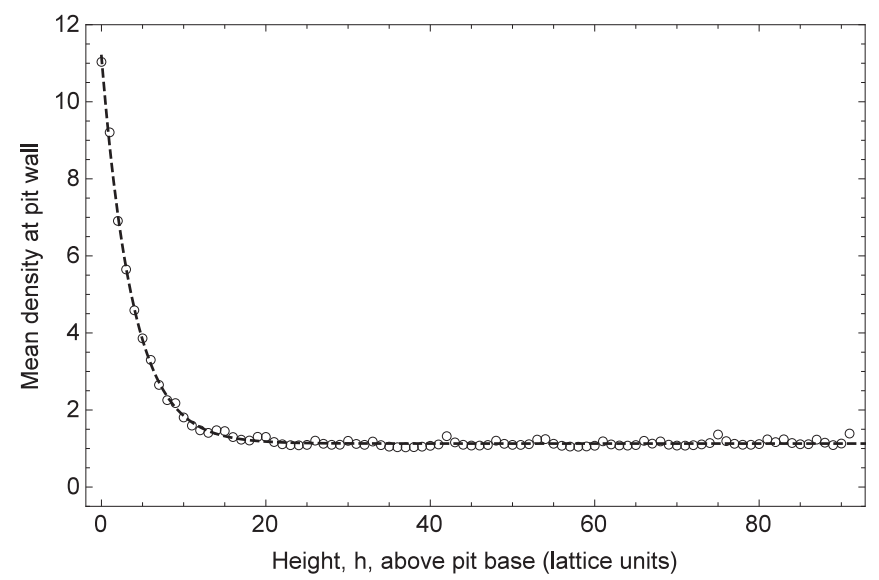

FIG. 10. Circles show mean particle density in sites adjacent to pit wall versus height above pit base for the central pit in Fig. 4 after $2 \times 10^{5}$ time steps. The model parameters are $S=10, \xi=0.05, b=$ $0.58, p_{c}=0.025, \rho=1$. The dashed line shows the function $A+$ $B \times \beta^{h}$, where $A=1.1, B=10.1, \beta=0.77$. shape depends only on this and the corrosion probability $p_{c}$, then we can expect to at least qualitatively capture the evolution of single pits.

\section{A. Definition of corrosion gradient model}

We define a basin or pit to be single local minimum in a surface (or a line or plane of minima) together with the set of surrounding points which may be connected to the minimum by surface trajectories which do not pass through any maxima or saddle points. We assume that the corrosion rate at a given point depends only on the height of that point relative to the minimum of the basin to which it belongs. We refer to this as a "corrosion gradient model." We define corrosion rate $r(h)$ per site face at relative height $h$ to be

$$
r(h)=1+\alpha \beta^{h},
$$

with $\beta \in[0,1]$ and $\alpha>0$. This simplification of the fitting form used in Fig. 10 is physically justified since corrosion rate may be rescaled by an overall constant by adjusting initial site strength. We let pits evolve in a similar way to the full model: At each time step the strength of exposed site $\vec{x}$ is reduced by

$$
\Delta S(\vec{x})=\sum_{\vec{y} \in G(\vec{x})} r[h(\vec{x})],
$$

where $h(\vec{x})$ is the height of site $\vec{x}$ relative to the minimum of its basin. As in the full model, all solid sites possess an initial strength uniform on $[(1-\xi) S,(1+\xi) S]$ for some choice of $S$ and $\xi<1$, and are removed from the solid immediately after their strength becomes negative.

In Fig. 11 we show a series of detailed snapshots of a surface which begins with a single site removed. As the pit becomes deeper, its base becomes wider and flatter, and a series of shelves is formed. Within a given shelf, sites that are closer to the center of the pit will be weaker because they have been exposed to corrosion for a longer period. The sites composing a shelf therefore disappear in sequence radially outwards from the center of the pit.

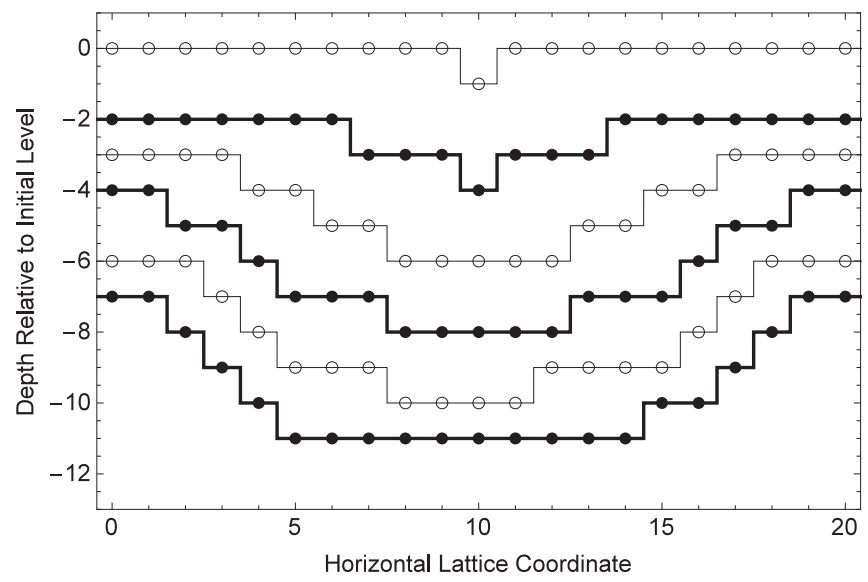

FIG. 11. Early stage evolution of a pit in the corrosion gradient model with $\alpha=1, \beta=0.5, S=50, \xi=0.01$. Here the surface is recorded after every 20 successive changes to the boundary. 


\section{B. Continuum evolution equation}

In the absence of randomness in the initial strengths of sites, the evolution of the surface is an entirely deterministic process. In this case numerical experiments show that the system finds a stable cycle of surface states. If the initial strengths of sites have nonzero variance $(\xi>0)$ then the evolution of the surface is a stochastic process whose randomness is derived from the particular configuration of site strengths. In this case the system finds similar, but transient, orbits whose persistence time is greater for smaller noise. By considering the life cycle of a typical site, we now derive an approximate evolution equation which is able to capture the form of these steady states.

When $\xi>0$ we define the random variable $H(x, t)$ to be the height, relative to pit base, of the uppermost face of the highest surface site at position $x$ at time $t$. We note that $H(x, t)$ is random as opposed to deterministic due to the random distribution of initial site strengths. For given $t$ the set of values of $H(x, t)$, one for each position $x$, describes the shape of the surface. The joint probability distribution of this set will depend on the initial configuration of the boundary between solid and gas sites and on the parameter $\xi$, which controls the variability in initial site strengths. We denote by $\mathbf{P}\{H(x, t)=h\}$ the (marginal [22]) probability that the height at position $x$ and time $t$ is equal to the particular value $h$. We then define $\eta(x, t)$ to be the expectation (average) of the random variable $H(x, t)$ over all possible realizations of random initial site strengths for given variance $\xi$, starting from a given initial state,

$$
\eta(x, t):=\sum_{h} h \mathbf{P}\{H(x, t)=h\}=\mathbf{E}[H(x, t)] .
$$

Here $\mathbf{E}$ denotes the expectation operator [22]. For given $t$, the set of values of $\eta(x, t)$, one for each position $x$, describes the average shape of the surface over all possible realizations of the distribution of site strengths. We focus on the case where $\xi$ is small enough to avoid disrupting regular pit structures.

We consider the case where the system evolves from an initial state where there is a unique lowest site in the surface, and we define its position to be the origin of coordinates; $x=0$. Without loss of generality we consider the shape of the wall which lies to the right $(x>0)$ of the base so that $H(x, t) \geqslant H(x-1, t)$. For given $x$, we define $\Delta H(x, t):=$ $H(x, t)-H(x-1, t)$ and note that the number of exposed faces at time $t$ is given by $1+\Delta H(x, t)$. We also define the discrete derivative of $\eta(x, t)$ with respect to $x$ :

$$
\eta^{\prime}(x, t):=\eta(x, t)-\eta(x-1, t) .
$$

We first consider the evolution of $\eta(x, t)$ when $\eta^{\prime}(x, t)$ is small and $\Delta H \in\{0,1\}$. Simulation results show that this is almost always the case provided we are sufficiently near the base of the pit. Under these conditions $\eta^{\prime}(x, t)$ is equal to the expected time that the surface site at position $x$ has two faces exposed, and $1-\eta^{\prime}(x, t)$ is the expected time that it has only one. If the highest site at position $x$ has a side face and an upper face exposed, then it will be exposed to corrosion rate $2+\alpha\left(\beta^{H-1}+\beta^{H}\right)$; if only its upper face is exposed, then its total corrosion rate will be $1+\alpha \beta^{H}$. Because the shelves of sites which form the low levels of the pit are destroyed in sequence radially out from the center, each surface site must h

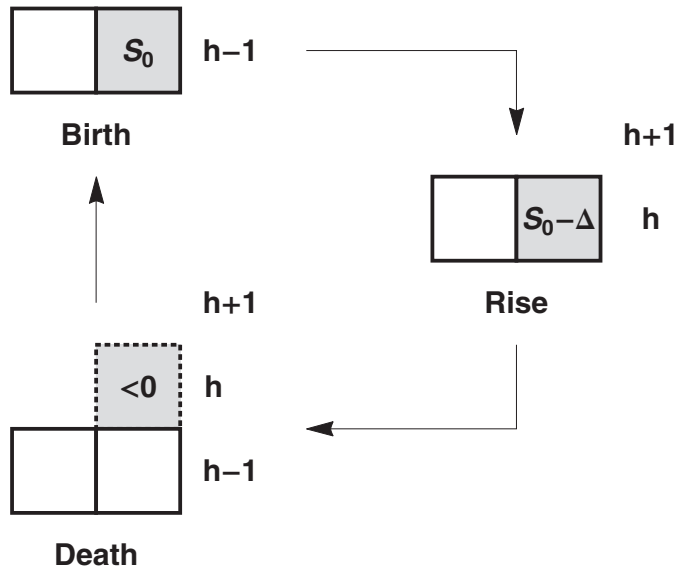

FIG. 12. The life cycle of a site (shaded, with initial strength $S_{0}$ ) near the base of the pit. The site is first exposed when $\Delta H=0$ (Birth); later its relative height increases (Rise). Its left neighbor will be destroyed before it so that it has two faces exposed prior to being destroyed (Death). The dotted boundary indicates a destroyed site (negative strength).

begin life with only its upper surface exposed and end life with two exposed faces. At some point during this life span the lowest site in the pit will be destroyed, increasing the relative height of all other sites. This cycle of events is illustrated in Fig. 12. Taking a weighted sum of the corrosion rates in the two possible $\Delta H$ states we arrive at the following approximate expression for the expected magnitude, $\Delta S$, of the change in surface strength per time step at $x$ :

$$
\begin{aligned}
\mathbf{E}[\Delta S] & \approx\left[1+\alpha \beta^{\eta}\right]\left(1-\eta^{\prime}\right)+\left[2+\alpha\left(\beta^{\eta}+\beta^{\eta+1}\right)\right] \eta^{\prime} \\
& =1+\alpha \beta^{\eta}+\left(1+\alpha \beta^{\eta+1}\right) \eta^{\prime} .
\end{aligned}
$$

Away from the base of the pit where $\eta \gg 0$ and $\Delta H$ can take larger values, it is no longer the case that $\eta^{\prime}(x, t)$ is the expected time for which two faces are exposed, so our derivation ceases to be valid. In this case we may write down a less sophisticated approximation for $\mathbf{E}[\Delta S]$ which does not require $\Delta H \in\{0,1\}$, but ignores the subtleties associated with the cycle illustrated in Fig. 12. Since the expected number of exposed faces at position $x$ is $1+\eta^{\prime}(x, t)$, then by neglecting differences in corrosion rates between the various exposed faces in position $x$, we have $\mathbf{E}[\Delta S] \approx\left(1+\eta^{\prime}\right)\left(1+\alpha \beta^{\eta}\right)$. This differs from our original approximation (8) by a quantity exponentially decaying with $\eta$. On the grounds that the $h$ dependent term in $r(h)$ is more significant near the pit base, we take Eq. (8) as our universal approximation for $\mathbf{E}[\Delta S]$.

In order to derive an expression for the discrete time derivative $\dot{\eta}(x, t):=\eta(x, t)-\eta(x, t-1)$ we must take account of a subtle but important correction which arises from the discrete nature of the model. Because the final change in site strength before a surface site is destroyed will, if $\xi>0$, certainly make the strength negative, then solid sites can absorb more units of corrosion than their initial strength value. They therefore have an effective strength in excess of their initial strength. If the final change in site strength has magnitude $\Delta S$, then the remaining strength will be approximately uniform on $[0, \Delta S]$ 
and therefore the expected effective strength of the site will be $S+\frac{1}{2} \Delta S$. For sites at level $H=0, \frac{1}{2} \Delta S=\frac{1}{2}(1+\alpha):=\epsilon_{1}$ and for all others $\frac{1}{2} \Delta S \approx \frac{1}{2}\left[2+\alpha\left(\beta^{\eta}+\beta^{\eta+1}\right)\right]:=\epsilon_{2}$. For a site with $\eta(x)>0$ and $x>0, \eta$ is increased by the corrosion of the pit base and decreased by corrosion events at position $x$ so that, provided $S \gg 1+\alpha$, for $x>0$,

$$
\begin{aligned}
\dot{\eta} & \approx \frac{1+\alpha}{S+\epsilon_{1}}-\frac{\mathbf{E}[\Delta S]}{S+\epsilon_{2}} \\
& \approx \frac{1+\alpha}{S}-\frac{\mathbf{E}[\Delta S]}{S}+\frac{1+\alpha}{S^{2}}\left(\epsilon_{2}-\epsilon_{1}\right) \\
& =\frac{1}{S}\left[\kappa_{0}-\kappa_{1} \beta^{\eta}-\left(1+\alpha \beta^{\eta+1}\right) \eta^{\prime}\right],
\end{aligned}
$$

where we have defined two constants,

$$
\begin{gathered}
\kappa_{0}=\alpha+\frac{(1+\alpha)(1-\alpha)}{2 S}, \\
\kappa_{1}=\alpha\left[1-\frac{(1+\beta)(1+\alpha)}{2 S}\right],
\end{gathered}
$$

and made use of the approximation $\mathbf{E}[\Delta S] \approx 1+\alpha$ in the order $S^{-2}$ term in order to obtain the intermediate equation (10). This relationship holds in equilibrium (when $\dot{\eta}=0$ ) because all positions must corrode the same rate. Out of equilibrium we are ignoring a correction of order $\mathcal{O}\left(S^{-2}\right)$ to the time derivative. The condition $x>0$ for the validity of Eq. (11) is an important one and arises because the site at $x=0$ forming the base of the pit must change in strength by $\Delta S=1+\alpha$ at every time step and has effective strength $S+\epsilon_{1}$ so that $\dot{\eta}(0, t)=0$.

Equation (11) is a difference equation in two variables and can only be solved numerically. However, we can extract analytical information if we interpret $\eta(x, t)$ as a function of continuous time and space variables $x, t$ so that (11) becomes a first order partial differential equation. Since $x=0$ is the deepest point of the pit, $\eta(0, t)=0$ for all $t \geqslant 0$ and we may find the steady state analytically, subject to this boundary condition, in implicit form:

$$
\eta(x)+\left(\frac{\kappa_{1}+\alpha \beta \kappa_{0}}{\kappa_{1} \ln \beta}\right) \ln \left[\frac{\kappa_{0}-\kappa_{1}}{\kappa_{0}-\kappa_{1} \beta^{\eta(x)}}\right]=\kappa_{0} x .
$$

Two examples of such steady states are plotted in Fig. 13 along with profiles obtained by simulating the corrosion gradient model. Figure 13 shows how the steady states of our approximate evolution equation closely match the simulation results and that the corrosion gradient model generates pits with the same trapezoidal shape found in the full model. From Eq. (14) we see that the gradient of the pit wall tends, for large $x$, to $\kappa_{0}$, which, for large $S$, is approximately equal to $\alpha$. In Sec. V we show how this gradient may be related to the parameters of the full model, by viewing each trapezoidal pit as a funnel which concentrates particles as they descend.

\section{Pit widths}

By making use of our implicit solution for the steady state pit profile we may derive an analytical expression for the width of its base. We define the edge of the pit as the solution to $\eta^{(3)}(x)=0$, which is the inflection point in the gradient of

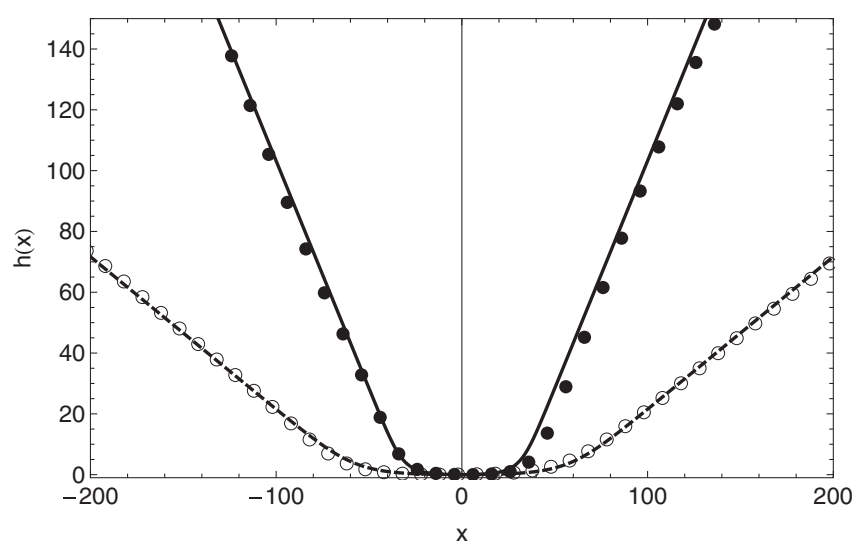

FIG. 13. Pit profiles in the corrosion gradient model, together with steady state solutions to the continuum evolution equation. Open circles show the case $\alpha=0.5, \beta=0.8, S=200$ and closed circles $\alpha=1.5, \beta=0.8, S=200$. In both cases the simulation results were obtained with $\xi=0.01$.

the wall. An implicit expression for $\eta^{(3)}(x)$ in terms of $\eta(x)$ may be obtained by repeatedly differentiating the steady state differential equation for $\eta(x)$. The condition $\eta^{(3)}(x)=0$ then reduces to a third order polynomial in $\beta^{\eta}$, having solution

$$
\beta^{\eta}=\frac{1+\alpha \beta-\sqrt{1+\alpha \beta+\alpha^{2} \beta^{2}}}{\alpha \beta}+\mathcal{O}\left(\frac{1}{S}\right) .
$$

Substitution into Eq. (14) gives an analytic expression for the width, $w$, of the pit base, which we provide here to lowest order in $S$ :

$$
\begin{aligned}
w:= & \frac{1}{\alpha \ln \beta} \ln \left[\frac{1+\alpha \beta-\sqrt{1+\alpha \beta+\alpha^{2} \beta^{2}}}{\alpha \beta}\right] \\
& +\frac{1+\alpha \beta}{\alpha \ln \beta} \ln \left[\frac{(1+\alpha)\left[1+\sqrt{1+\alpha \beta+\alpha^{2} \beta^{2}}\right]}{2 \alpha S}\right] \\
& +\mathcal{O}\left(\frac{\ln S}{S}\right) .
\end{aligned}
$$

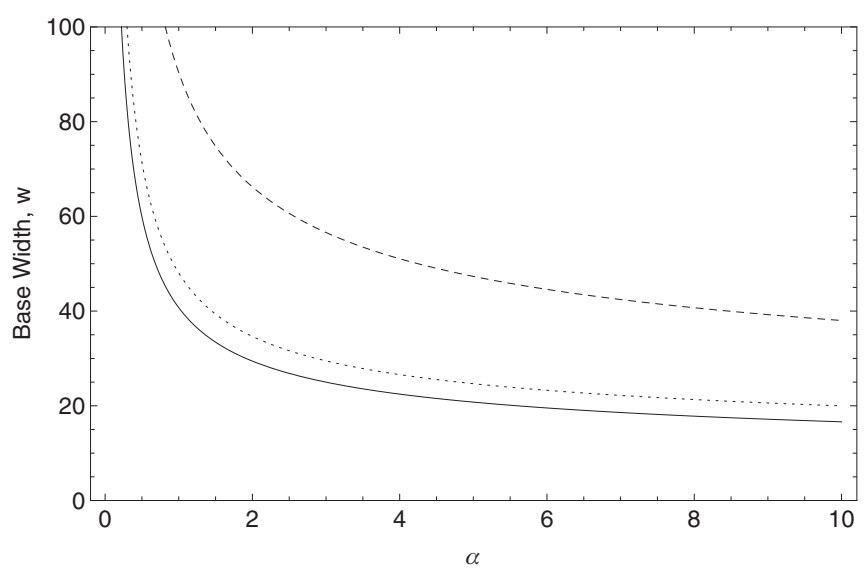

FIG. 14. Width of pit base versus $\alpha$ (equivalent to wall gradient in the limit $S \rightarrow \infty$ ). Parameter values are $\beta=0.8, S=500$ (dotted), $\beta=0.8, S=200$ (solid), and $\beta=0.9, S=200$ (dashed). 


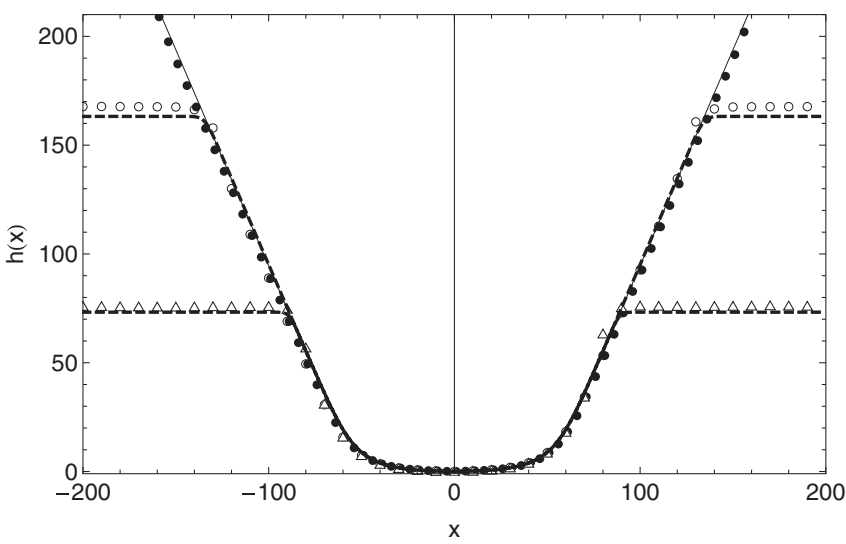

FIG. 15. Simulated pit profiles at times $t=5.53 \times 10^{3}$ (triangles), $t=1.01 \times 10^{4}$ (open circles), and $t=1.39 \times 10^{4}$ (solid circles). Parameter values are $\alpha=2.0, \beta=0.9, S=100, \xi=0.01$. The thick dashed lines give numerical solutions to Eq. (11) at the corresponding times, whereas the continuous line gives the steady state.

In the limit $\alpha \rightarrow 0$ both terms diverge in magnitude but with opposite signs, the second term being positive with a higher order divergence. Therefore, the pit width tends to infinity as $\alpha$ (the wall gradient) tends to zero. Narrower based pits will therefore have steeper walls. As $S \rightarrow \infty$ the second term possesses a divergence $\propto \ln S$, implying that harder surfaces produce wider pits. Both terms diverge as $\beta \rightarrow 1$. This limit is equivalent to the limit of zero downward bias. Figure 14 illustrates these effects.

\section{Formation dynamics}

We now turn to the dynamical process by which pits are formed, which is described approximately by Eq. (11). We interpret this as a partial differential equation in $x$ and $t$, but note that because of the condition $\dot{\eta}(0, t)=0$ it is not analytically tractable. We solve the equation numerically for $x \geqslant 0$ using the method of lines [23] from an initial condition $\eta(x, 0)=0$. In Fig. 15 we see that, as the pit deepens, the internal structure which has already formed is preserved. While in this example the pit can continue to deepen indefinitely, in the full model the presence of spontaneously formed neighboring pits limits their depth.

\section{TRAPEZOIDAL FUNNEL EFFECT}

We may find an approximate relationship between the parameter $\alpha$ of the corrosion gradient analysis and the parameters $b, p_{c}$ by considering the stability of a trapezoidal pit in the surface of the full model. Because particles are biased downwards, the pit acts like a funnel which concentrates the particles into a narrower space as they descend. However, the funnel has absorbing sides which counteract this effect. If these two effects are not in balance at the mouth of the funnel, then they will tend toward a state of balance at lower levels because increasing particle concentration leads to an increase in absorbtion rate. However, changes in corrosion rate with depth will distort the constant wall gradient over time, making the trapezoidal geometry unstable. Therefore, in order for a trapezoidal pit to be stable, funneling and absorption must be in balance at the mouth of the pit.

Given that we expect particle density near the wall of a stable trapezoidal pit to be equal at all levels, then if particle density is approximately constant across the pit mouth, it must remain so at lower levels. This observation leads to an analytical approximation for stable pit gradient, derived using simple random walks. The expression is approximate because the presence of peaks in the pit structure and discrete steps in the pit wall distort the particle density at the mouth and adjacent to the walls. We take account of these effects in a more technical but less tractable calculation.

\section{A. Constant density approximation using simple random walk}

The net downward drift of a particle in the vicinity of a sloping pit wall will tend to bring it closer to the wall. If the gradient of the wall is $m$, then in a reference frame with its origin at the wall, but moving so as to remain level with the particle, the particle will appear to have a net velocity toward the wall,

$$
v=\frac{2 b-1}{2 m} .
$$

We assume that $m \geqslant 1$ so that the horizontal motion in this reference frame is a discrete time simple random walk [24]. We approximate this walk as uncorrelated with

$$
\mathbf{P}(\text { no step })=\frac{m-1}{2 m},
$$

which is the fraction of steps in two dimensions which do not change the horizontal distance between the particle and the wall, accounting for the fact that the wall is composed of vertical faces. We also assume that the pit is sufficiently wide so that the influence of the opposite wall can be neglected and with effectively infinite depth so that we need not consider the influence of the base. Letting $a$ be the probability of a move toward the wall, with the probability of remaining still given by (18), then the correct net velocity (17) is obtained if

$$
a=\frac{2 b+m}{4 m} .
$$

Let $\phi_{k}$ be the equilibrium particle density $k$ steps away from the position $(k=0)$ immediately adjacent to the wall then for $k>0$ :

$$
(2 b+m) \phi_{k+1}-2(m+1) \phi_{k}+(2-2 b+m) \phi_{k-1}=0 .
$$

A fraction $1 / m$ of the sites adjacent to the wall are corner sites since they they are bordered by two wall sites, one to the side and one below. We assume that conditional on a particle being adjacent to the wall, the probability that it occupies a corner site is $1 / \mathrm{m}$. In this case the probability that a particle which is adjacent to the wall will attempt to jump into it is $(m+2 b) / 4 m$. Given that $p_{c}$ is the probability of absorption if a particle attempts to step into the wall, then the following boundary condition holds:

$$
\left[m+2(1-b)+(2 b+m) p_{c}\right] \phi_{0}=[2 b+m] \phi_{1} .
$$


Since we are treating our pit as having effectively infinite width then, as $k \rightarrow \infty$ the particle density must tend to its value at height $h_{G}$ (see Sec. III) above the surface: $\rho=1$. We therefore require that $\lim _{k \rightarrow \infty} \phi_{k}=1$. Solving Eq. (20) under these conditions we find that

$$
\phi_{k}=1+c\left[\frac{1+m(1-2 a)}{2 a m}\right]^{k},
$$

where

$$
c=\frac{2(2 b-1)-(2 b+m) p_{c}}{(2 b+m) p_{c}} .
$$

We approximate the density profile at the mouth of the pit with its value above the surface. If the pit gradient is stable, we expect the equilibrium profile to match this: $\phi_{k}=1$ for all $k \geqslant 0$ so $c=0$. Imposing this condition we find that

$$
m\left(b, p_{c}\right)=\frac{2\left[\left(2-p_{c}\right) b-1\right]}{p_{c}},
$$

which is the approximate gradient at which the particle density across the pit will remain constant, until the influence of the base or the interaction between the two opposing walls becomes significant.

\section{B. Simulated wall gradient}

We have estimated the wall gradient as a function of $p_{c}$ for various fixed bias values by simulating a surface in a $d=2$ system, where the the corrosion probability varies slowly and continuously with position. In order to relate wall gradient to position, we compute the set, $\mathcal{D}_{1}(x, N)$, of finite difference first derivatives in a window of width $2 N+1$ for some $N>0$ centered on position $x$

$$
\mathcal{D}_{1}(x, N):=\{H(k)-H(k-1)\}_{k=x-N}^{x+N} .
$$

The constant gradient sections of wall are the steepest parts of the surface, and provided that the window is significantly wider than the pit base, then each window will contain such a section. The wall gradient may then be estimated as the mean of the largest $n$ elements of $\mathcal{D}_{1}(x, N)$ with $n$ small enough so that all $n$ elements belong to a constant gradient section. In Fig. 16 this process has been used to estimate wall gradients with $N=25$ and $n=10$ for $b \in\{0.6,0.75\}$. Also shown in Fig. 16 are graphs of $m\left(b, p_{c}\right)$ defined in Eq. (24), which is our estimated gradient if the particle density were equal to unity across the mouth of the pit. We see that while (24) is a close approximation when $b=0.6$, the approximation is poorer when $b=0.75$. We now address this point.

\section{Approximation using exact equilibrium density profile near a sloping wall}

The presence of peaks in the pitted surface distorts the particle field at the mouths of the pits. Regions of increased density appear immediately above the peaks where particles collect before entering the pit (Fig. 17). Also, the discrete nature of the surface creates steps in the pit wall above which the particle density is increased. We ignored these effects in our simple analysis by assuming that particle density is constant across the pit mouth, and that all sites adjacent to the pit wall have equal particle density. These approximations can be

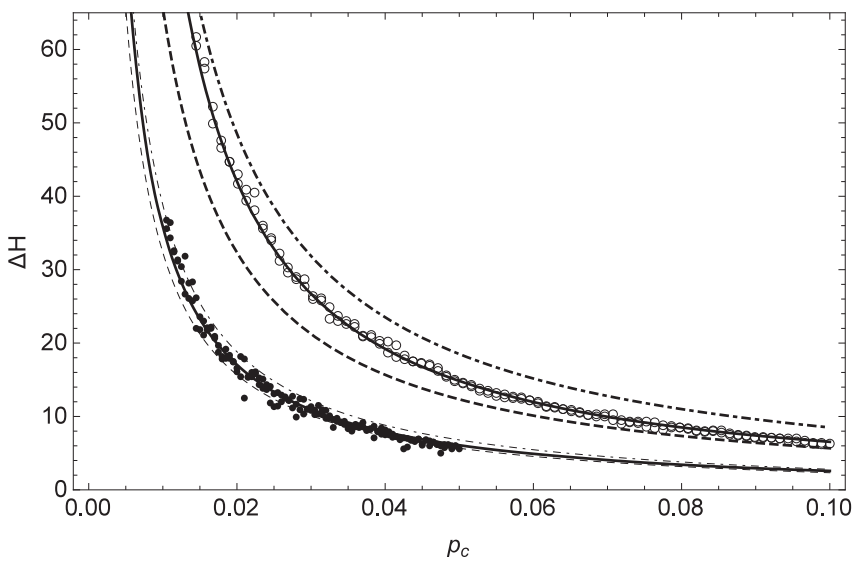

FIG. 16. Open circles show estimated wall gradients (defined in main text) versus $p_{c}$ in a system of length 4000 , where $p_{c} \in[0.01,0.1]$ and $b=0.75$. Dots show estimated wall gradients versus $p_{c}$ in a system of length 4000 , where $p_{c} \in[0.01,0.05]$ and $b=0.6$. The dotdashed lines are graphs of $m\left(b, p_{c}\right)$ defined in Eq. (24) for $b=0.75$ (thick line) and $b=0.6$ (thin line). The dashed lines are graphs of $\gamma\left(b, p_{c}\right)$ [Eq. (34)] for $b=0.75$ (thick line) and $b=0.6$ (thin line). The solid lines are the sloping wall approximations described in Sec. V C.

improved upon using an exact calculation of the equilibrium particle density near the stepped wall.

We consider a single step in an infinite sloping wall with gradient $m \in\{1,2,3, \ldots\}$. Let the particle density in the site immediately above the step be $\pi_{0}$ and the density $k$ steps above that be $\pi_{k}$. Figure 18 illustrates the case where $m=4$. If the particle density is in equilibrium, then the column of sites above every step must have an identical density profile, as shown in Fig. 18. In sites not adjacent to the wall, having indices $k \geqslant m$, we have

$$
\pi_{k+m}+2 b \pi_{k+1}-4 \pi_{k}+2(1-b) \pi_{k-1}+\pi_{k-m}=0 .
$$

The general solution to this equation [25] may be written in terms of the roots $\left\{\lambda_{1}, \ldots, \lambda_{2 m}\right\}$ of the characteristic polynomial

$$
\lambda^{2 m}+2 b \lambda^{m+1}-4 \lambda^{m}+2(1-b) \lambda^{m-1}+1=0 .
$$

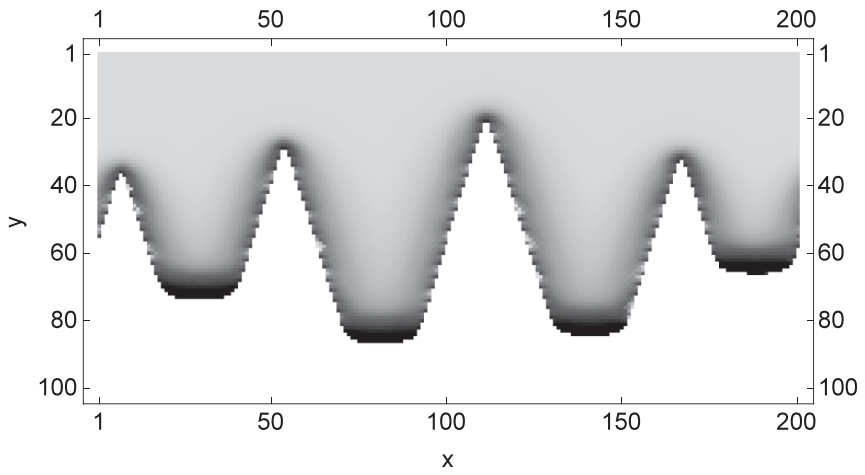

FIG. 17. Plot of the particle density field where higher density is darker gray. $x$ and $y$ are horizontal and vertical lattice coordinates. Parameter values are $p_{c}=0.1, b=0.65, S=10, \xi=0.05$. 


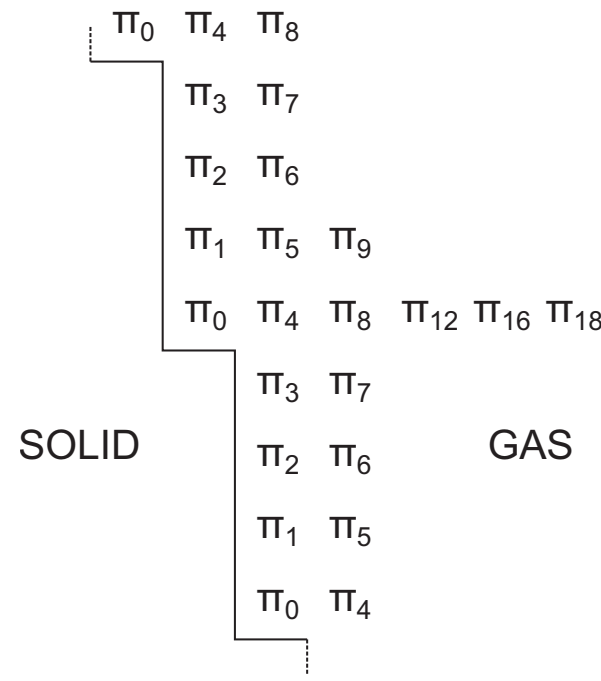

FIG. 18. Schematic representation of the equilibrium density distribution adjacent to a discrete sloping wall of gradient $m=4$. The variables $\pi_{i}$ are particle densities.

We assume the roots to be ordered by absolute value so that $\left|\lambda_{k+1}\right| \geqslant\left|\lambda_{k}\right|$. If $\lim _{k \rightarrow \infty} \pi_{k}=1$ then only roots with $\left|\lambda_{k}\right| \leqslant 1$, of which there are $m+1$, the largest of which is $\lambda_{m+1}=1$, can contribute to the solution

$$
\pi_{n}=1+\sum_{k=1}^{m} c_{k} \lambda_{k}^{n}
$$

The constants $c_{k}$ must be found using the boundary conditions for sites adjacent to the wall,

$$
\begin{gathered}
\left(3+p_{c}\right) \pi_{k}-\pi_{k+m}-2 b \pi_{k+1}-2(1-b) \pi_{k-1}=0, \\
{\left[3-2 b+(2 b+1) p_{c}\right] \pi_{0}-\pi_{m}-2 b \pi_{1}=0,}
\end{gathered}
$$

where (29) holds for $k \in\{1, \ldots, m-1\}$. An example of this solution is shown in Fig. 19.

We note that the solution oscillates with period $m$, matching the period of steps in the wall.

Also shown in Fig. 19 is the density in a column of sites above a step in a spontaneously formed sloping wall in the full model with the same $b, p_{c}$ values that were used in the exact solution (the full model was simulated first and the value $m=10$ in the exact solution chosen to match the spontaneously formed wall gradient). Although the average particle density near the wall is much larger than the bulk value $(\rho=1)$, the minimum of each oscillation is approximately equal to this value. This suggests that an appropriate condition on the exact solution required to relate $m$ to $b$ and $p_{c}$ is $\min \left\{\pi_{0}, \pi_{1}, \ldots, \pi_{m-1}\right\}=1$. For a given value $b$, we may determine the relationship between $m$ and $p_{c}$ by finding the value of $p_{c}$ for which this condition holds. The solid curves in Fig. 16 were obtained by this method, and we see that the gradient estimates are accurate. We note, however, that it is not possible to write an analytical relation expression for $m$.

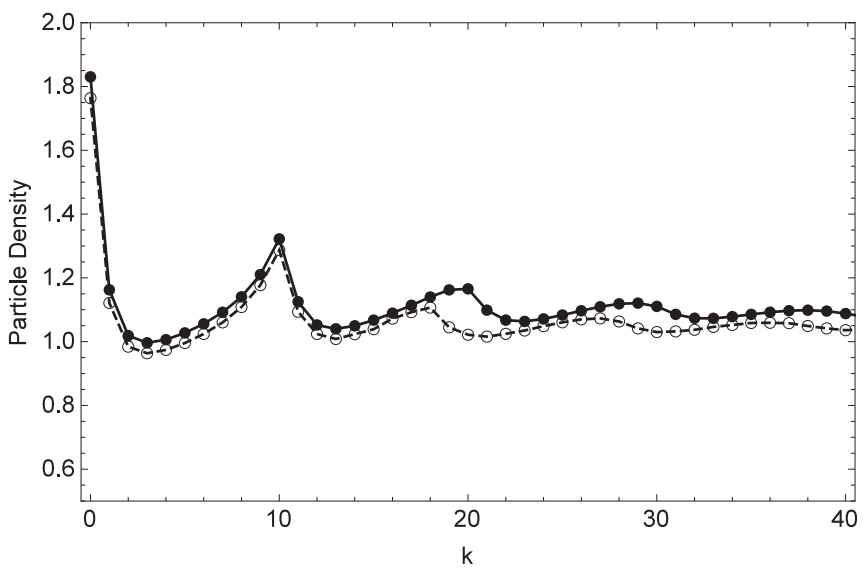

FIG. 19. Solid circles with solid line show the exact equilibrium particle density in a column above a step in a discrete sloping wall with gradient $m=10$ and $b=0.75, p_{c}=0.07$. Open circles with dashed line shows the particle density in a column above a step in a spontaneously formed sloping wall in the full model with $b=$ $0.75, p_{c}=0.07$.

\section{Connection to corrosion gradient analysis}

Our theoretical estimates of the wall gradient provide a link between the full model and the corrosion gradient model. In this latter model we found that the wall gradient is approximately equal (with corrections of order $1 / S$ ) to the constant $\alpha$ in the corrosion rate function $r(h)=1+\alpha \beta^{h}$ [Eq. (4)]. The trapezoidal funnel effect also explains why the particle density, and therefore the corrosion rate, is approximately constant adjacent to the pit walls. It remains to interpret the constant $\beta$ in terms of the full model. Although we cannot provide a precise relationship between $\beta, b$, and $p_{c}$, insight into both wall gradients and $\beta$ may be gained by considering the particle density above the base of the pit. We have argued that particles should neither be concentrated nor depleted as we descend into the pit, so we expect the particle density above the flat base to be independent of the pit depth and therefore to take a similar form to the equilibrium particle density above a flat, partially absorbing surface. Considering only the vertical motion of a particle above such a surface in the full model and letting $\psi_{k}$ be the equilibrium density $k$ steps above the site adjacent to the surface we have

$$
\begin{gathered}
\psi_{k}=b \psi_{k+1}+(1-b) \psi_{k-1}, \\
b \psi_{1}=\left[1-\left(1-p_{c}\right) b\right] \psi_{0},
\end{gathered}
$$

with $\lim _{k \rightarrow \infty} \psi_{k}=1$. Solving for $\psi_{k}$ we find that

$$
\psi_{k}=1+\left[\frac{\left(2-p_{c}\right) b-1}{b p_{c}}\right]\left(\frac{1-b}{b}\right)^{k} .
$$

We note the similarity between the wall gradient approximation (24) and the coefficient of the exponential term in (33), which we define as a new function,

$$
\gamma\left(b, p_{c}\right)=\frac{\left(2-p_{c}\right) b-1}{b p_{c}} .
$$


In the limit $b \rightarrow \frac{1}{2}$ the functions $\gamma\left(b, p_{c}\right)$ and $m\left(b, p_{c}\right)$ become identical. The function $\gamma\left(b, p_{c}\right)$ is graphed in Fig. 16 and we see that, although it underestimates the wall gradient, the error is of similar magnitude to Eq. (24). This suggests Eq. (33) as a crude approximation to the corrosion rate function (4) so that $\beta \approx(1-b) / b$. For the parameter values used in Fig. 10 we found by regression that $\beta \approx 0.77$. In that case the bias was $b=0.58$, which gives $(1-b) / b=0.72$. We have also computed $\beta$ by regression using the density profile in Fig. 17, finding that $\beta \approx 0.58$. In that case, $b=0.65$, which gives $(1-b) / b=0.54$.

\section{RELATIONSHIP TO EXPERIMENT}

Experimental work on rock weathering has focused on the role of specific agents and, in particular, the action of salts [26,27]. Attempts have been made to produce regular pit formations in rock under laboratory conditions [13], and a few pits were produced, but no coordinated system of hollows. The limited experimental work in this area may reflect the difficulty in identifying the key parameters involved in tafoni formation and in controlling the interactions between them. One recent attempt to explore such interactions [28] looked at the effect on pore characteristics produced by salt agents. A further challenge to reproducing natural formations in the laboratory is the time taken for natural rock formations to develop, which ranges from decades to millennia [15]. Experiments have tended to focus on conditions that will produce visible and measurable changes in form over a few months, using highly aggressive salt treatments and extreme temperature variations [29].

Our model suggests that gradients in the concentration of aggressive material, or in other physical conditions which accelerate damage, such as humidity [30-32], are crucial to the development of pits. We have also seen that excessively aggressive agents or absorbent surfaces (high $p_{c}$ values in our model) produce shallower pits and can prevent pit formation altogether. Steep concentration gradients, created by diffusion bias in the model, are predicted to produce narrower pits with steeper walls. The fact that pit width, wall steepness, and concentration gradient appear to possess approximate analytical relationships within the model suggests that comparing field data on pit geometry and concentration gradients may be fruitful in revealing underlying relationships in nature. Our model could be tested in the laboratory using a simple erosive agent, for example, a salt spray, and a rock of relatively homogeneous strength (or an artificial material such as plaster of Paris) to provide a simple surface. Salt sprays have been used previously to simulate the action of sea spray upon a rock surface [27]. For a spray released above a horizontal surface, we would expect smaller droplets to result in lower concentration gradients due to the domination of diffusion over gravity induced bias. In this way concentration gradients could be artificially varied. In addition, we would expect the absorption probability $p_{c}$ of the model to be related to the pore structure and absorbency of the surface. We emphasize, however, that the creation of regular pit formations in rock surfaces under laboratory conditions remains an open challenge.
The model may also inform experimental work on the dynamics of the early stages of pit formation. Our simulations suggest that for the formation of tafoni to begin we require a certain minimum level of inhomogeneity in surface strength and a balance between corrosive power and bias. As our simulations show, regular pit structures emerge from random fluctuations in the surface caused by erosion. This suggests that the early stages of tafoni development might be identified through a detailed examination of changing surface morphology. We expect that initially surface depressions will form and then slowly coalesce. Detailed surface photography or close-range laser scanning techniques could be used to visually identify the development of such depressions [33]. It would be expected that the experimental examination of the development of tafoni would require months of experimental weathering, perhaps in the range of the 24000 erosive cycles [29]. The ability to distinguish between early surface fluctuations which lead to pit formation as opposed to simple surface lowering would provide a mechanism for determining the salt concentrations, bias, and strength inhomogeneity required to allow pit formation to begin. It would also allow experimental verification of the depth dynamics predicted by the model.

The model may also have relevance to experiments on the corrosion of metals, although here the link between the features of our model and the chemistry at play in metal corrosion is more subtle. Pitting typically occurs when a solution containing chloride ions (the corrosive particle) comes into contact with a metal surface. The pits are found to have high concentrations of chloride within them, which sustains further pit growth. The conventional explanation of how these concentrations develop is the formation of a potential difference between the mouth and the interior of the pit which draws anions (chloride ions) into the pit [34]. However, it has been suggested [2] that the high concentration of metal cations in the pit, which can only escape by diffusion, is the controlling factor for the concentration of chloride anions, and not the potential drop. In either case there is a current of diffusing corrosive particles drawn into the pit, and this behavior is captured by the biased diffusion of the particles in our model. An important additional factor present in metal pitting is passivation, where a protective oxide layer forms on the surface and pit formation is highly localized where this protective layer breaks down (the "initiation stage" of pit formation). This effect is not captured in our model, but our analysis of the propagation of a single pit is relevant and provides approximate analytical relationships between the pit geometry and potential drop, studied previously by simulation [17]. Image analysis of pits formed in aluminum by the action of sodium chloride [35], showed that $\approx 86 \%$ of "regular" pits possessed rectangularity [36], $R$ (ratio of the area of pit cross section to the area of the minimum bounding rectangle of this cross section), in the interval [0.5,0.72], where $R=0.5$ represents a cone, and $R>0.72$ represents approximately hemispherical pits. These results are consistent, for a range parameter values, with the trapezoidal geometry $R>0.5$ found in our model. The regular formations seen in our model may be relevant in surface regions where the passive layer has completely broken down and clusters of pits have formed $[16,35]$. 


\section{DISCUSSION AND CONCLUSION}

We have introduced a simple lattice model of surface damage by incident particles in which pits spontaneously form in one and two dimensional surfaces. Surface damage is caused by corrosive material which diffuses onto the surface, having some fixed probability of absorbtion at each contact. Our simplified model demonstrates how large scale, regular pit formations can arise spontaneously from microscopic rules. Such formations have been observed in rocks [1,14,15,37] and to some extent in metals [16,35]. The model also provides insight into field observations of the growth rate of pits [15] and provides approximate analytical relationships between parameters describing the geometry of pits, corrosion gradients, and the hardness and inhomogeneity of the surface.

Essential to the formation of pit structures in the model is the presence concentration gradients of aggressive material, brought about by a bias in the diffusing particles. Analysis of the microclimates in tafoni show the presence of gradients in moisture [30-32,38] and salt [39] concentrations from the mouth of pits to their bases. Both salt concentration and moisture (measured as relative humidity) increase from the mouth to the base. The sheltering effect of pits [40] may reduce the evaporation of water, contributing to the humidity gradient. Combined these observations suggest that, once established, the tafoni produce a protective environment which stabilizes the internal moisture and salt concentration gradients, creating positive feedback on further development.

An analytical theory for the spontaneous regular arrangement of pits within our model remains to be found. The fluctuations, seen in Fig. 3, in the early stages of the formation process may be viewed as a superposition of multiple protopits, most of which are absorbed into other larger pits. Two effects appear important to the arrangement process. First, there is a critical depth at which pits become stable in the sense that a large neighboring pit will not absorb them. Below this depth pits can coagulate. Second, pits appear to be able to migrate small distances across the surface. We conjecture that the combination of migration and coagulation is responsible for regularity in the final arrangement.

We note finally that we have explored only one form of corrosion rate function in our corrosion gradient analysis. Alternative definitions of this function have the potential to allow us to explore corrosion processes other than diffusion and absorption of corrosive particles, provided the corrosion rate is a known function of height relative to pit base.

\section{ACKNOWLEDGMENTS}

The authors would like to thank the anonymous referees for their useful comments and also Samia Burridge for careful proofreading and discussion of this work.
[1] G. E. Mustoe, Geol. Soc. Am. Bull. 93, 108 (1982).

[2] G. T. Burstein, C. Liu, R. M. Souto, and S. P. Vines, Corros. Eng., Sci. Technol. 39, 25 (2004).

[3] A. V. Turkington and J. D. Phillips, Earth Surf. Processes Landforms 29, 665 (2004).

[4] R. U. Cooke, A. Warren, and A. Goudie, Desert Geomorphology (UCL Press, London, 1993).

[5] Y. Matsukura and N. Matsuoka, Earth Surf. Processes Landforms 16, 51 (1991).

[6] J. L. Conca and A. M. Astor, Geology 15, 151 (1987).

[7] G. E. Mustoe, Earth Surf. Processes Landforms 35, 424 (2010).

[8] H. A. Viles, Earth Surf. Processes Landforms 30, 1471 (2005).

[9] E. F. McBride and M. D. Picard, Earth Surf. Processes Landforms 29, 713 (2004).

[10] L. Mol and H. A. Viles, Earth Surf. Processes Landforms 37, 301 (2012).

[11] D. N. Mottershead and K. Pye, Earth Surf. Processes Landforms 19, 543 (1994).

[12] J. L. Conca and G. R. Rossman, J. Geol. 93, 59 (1985).

[13] C. R. Rodriguez-Navarro, E. Doehne, and E. Sebastian, Geol. Soc. Am. Bull. 111, 1250 (1999).

[14] H. P. Huinink, L. Pel, and K. Kopinga, Earth Surf. Processes Landforms 29, 1225 (2004).

[15] T. Sunamura and H. Aoki, Earth Surf. Processes Landforms 36, 1624 (2011).

[16] R. M. Pidaparti, L. Fang, and M. J. Palakal, Comput. Mater. Sci. 41, 255 (2008)

[17] B. Malki and B. Baroux, Corros. Sci. 47, 171 (2005).

[18] J. Stafiej, D. di Caprio, and L. Bartosik, J. Supercomput. 65, 697 (2013).
[19] A. L. Barabasi and H. E. Stanley, Fractal Concepts in Surface Growth (Cambridge University Press, Cambridge, UK, 1995).

[20] N. G. Van Kampen, Stochastic Processes in Physics and Chemistry (Elsevier, Amsterdam, 2007).

[21] S. A. Norwick and L. R. Dexter, Earth Surf. Processes Landforms 27, 11 (2002).

[22] G. Grimmett and D. Stirzaker, Probability and Random Processes (Oxford University Press, Oxford, UK, 2001).

[23] W. E. Schiesse and G. W. Griffiths, A Compendium of Partial Differential Equation Models: Method of Lines Analysis with Matlab (Cambridge University Press, Cambridge, UK, 2009).

[24] G. F. Lawler, Introduction to Stochastic Processes (Chapman \& Hall, London, New York, 2006).

[25] S. Goldberg, Introduction to Difference Equations (Dover, New York, 1986).

[26] R. B. G. Williams and D. A. Robinson, Earth Surf. Processes Landforms 26, 811 (2001).

[27] C. Cardell et al., Earth Surf. Processes Landforms 28, 1 (2003).

[28] O. Buj, J. Gisbert, J. M. McKinley, and B. Smith, Earth Surf. Processes Landforms 36, 383 (2011).

[29] B. J. Smith, P. A. Warke, J. P. McGreevy, and H. L. Kane, Geomorphology 67, 211 (2005).

[30] B. J. Smith, Z. Geomorphol. 22, 21 (1978).

[31] I. P. Martini, Z. Geomorphol. 22, 44 (1978).

[32] D. Dragovich, Z. Geomorphol. 13, 163 (1969).

[33] M. Schaefer and R. Inkpen, Earth Surf. Processes Landforms 35, 147 (2010).

[34] R. W. Revie and H. H. Uhlig, Corrosion and Corrosion Control (Wiley-Interscience, New Jersey, 2008). 
[35] J. W. J. Silva, A. G. Bustamante, E. N. Codaro, R. Z. Nakazato, and L. R. O. Hein, Appl. Surf. Sci. 236, 356 (2004).

[36] L. da Fontoura Costa and R. Marcondes Cesar, Shape Classification and Analysis (CRC Press, Boca Raton, FL, 2009).

[37] J. F. McCauley, C. S. Breed, F. El-Baz, M. I. Whitney, M. J. Grolier, and A. W. Ward, J. Geophys. Res. 84, 8222 (1979).
[38] A. Mellor, J. Short, and S. J. Kirkby, Earth Surf. Processes Landforms 22, 817 (1997).

[39] G. E. Mustoe, Earth Surf. Processes Landforms 8, 517 (1983).

[40] H. Wang, E. S. Takle, and J. Shen, Annu. Rev. Fluid Mech. 33, 549 (2001). 